\title{
An Improved Particle Swarm Optimization Algorithm Mimicking Territorial Dispute Between Groups for Multimodal Function Optimization Problems
}

\author{
Jang-Ho Seo ${ }^{1}$, Chang-Hwan Im² ${ }^{2}$ Sang-Yeop Kwak ${ }^{1}$, Cheol-Gyun Lee ${ }^{3}$, and Hyun-Kyo Jung ${ }^{1}$ \\ ${ }^{1}$ School of Electrical Engineering and Computer Science, Seoul National University, Kwanak-gu, Seoul 151-744, Korea \\ ${ }^{2}$ Department of Biomedical Engineering, Yonsei University, Kangwon-do 220-710, Korea \\ ${ }^{3}$ Department of Electrical Engineering, Dong-Eui University, Pusan, Korea
}

\begin{abstract}
In the present paper, an improved particle swarm optimization (PSO) algorithm for multimodal function optimization is proposed. The new algorithm, named auto-tuning multigrouped PSO (AT-MGPSO) algorithm mimics natural phenomena in ecosystem such as territorial dispute between different group members and immigration of weak groups, resulting in automatic determination of the size of each group's territory and robust convergence. The usefulness of the proposed algorithm is verified by the application to a specially designed test function and a practical electromagnetic optimization problem.
\end{abstract}

Index Terms-Electromagnetic optimization problems, multi-grouped particle swarm optimization (MGPSO), multimodal function optimization, particle swarm optimization (PSO).

\section{INTRODUCTION}

$\mathbf{P}$ ARTICLE SWARM optimization (PSO) is a kind of stochastic optimization algorithms proposed by Kennedy and Eberhart [1]. Originally, PSO was inspired by the sociological behavior associated with birds flocking. In the original version of PSO, particles fly through the search space influenced by two factors: each individual's best position ever found (pbest) and the group's best position ever found (gbest). Due to its simple mechanism and high performance in global optimization, PSO has been successfully applied to many optimization problems [2]-[4]. Moreover, this new evolutionary computation technique, based on the movement and intelligence of swarms, has shown better convergence characteristics than conventional stochastic optimization methods such as genetic algorithms (GAs) and simulated annealing [1], [4].

In recent years, there have been several attempts to apply PSO to multimodal function optimization problems [5]-[7], which find more than one optimum of a multimodal function. In our previous study [7], we have proposed a new algorithm named multi-grouped PSO (MGPSO) to apply PSO to the multimodal function optimization problems. Contrary to the conventional attempts for the multimodal PSO, MGPSO used multiple groups which tracks their own best solution independently and thus guaranteed that one can search $N$ 'superior' peaks of a multimodal function when the number of groups is $N$. In MGPSO, we gave each gbest a 'territory' which prevents other solutions from intruding and introduced a concept called repulsive velocity, in order to avoid overlapping of the discovered solutions. This new approach, however, had a potential problem in that the initial size of each group's territory was set as an identical value and the size was decreased as iteration number increased. Therefore, if the size of the territory became too small before sufficient convergence level, some groups could not find their own peaks and wandered around other groups' gbests.
In the present paper, a new algorithm named auto-tuning MGPSO (AT-MGPSO) is proposed in order to alleviate this problem and guarantee robust convergence regardless of the territory size. To implement AT-MPGSO, we have mimicked herd instinct in ecosystem such as territorial dispute between different groups and immigration of weak groups.

\section{CONVENTIONAL MGPSO}

In our previous study [7], we introduced a new concept named repulsive velocity to encourage individual particles, located in territory of the gbest of other group, to escape from the other groups' territory in a more efficient manner. The velocity of a particle was then updated according to the following:

$$
\begin{aligned}
& \mathbf{V}_{\mathbf{i j}}^{\mathbf{k}+\mathbf{1}}=w \mathbf{V}_{\mathbf{i j}}^{\mathbf{k}}+C_{1} \varphi_{1}\left(\text { pbest }_{\mathbf{i j}}^{\mathbf{k}}-\mathbf{X}_{\mathbf{i j}}^{\mathbf{k}}\right) \\
& \quad+C_{2} \varphi_{2}\left(\text { gbest }_{\mathbf{i}}^{\mathbf{k}}-\mathbf{X}_{\mathbf{i j}}^{\mathbf{k}}\right)+C_{3} \varphi_{3}\left(\mathbf{X}_{\mathbf{i j}}^{\mathbf{k}}-\text { gbest }_{\mathbf{m}}^{\mathbf{k}}\right)
\end{aligned}
$$

where $\mathbf{V}_{\mathbf{i j}}^{\mathbf{k}}$ and $\mathbf{X}_{\mathbf{i j}}^{\mathbf{k}}$ are velocity and position of $\mathbf{j}$ th particle in ith group at kth iteration, respectively. pbest $_{\mathbf{i j}}^{\mathbf{k}}$ is pbest of $\mathbf{j}$ th particle in ith group and $\mathbf{g}_{\text {best }_{\mathbf{i}}^{\mathbf{k}}}$ is gbest of ith group. $C_{3}$ is the repulsive coefficient, which has a zero value if the $\mathrm{jth}$ particle does not intrude a territory of other group's gbest. gbest $_{\mathbf{m}}^{\mathbf{k}}$ is the gbest of other group (mth group), of which the territory is intruded by the $\mathrm{jth}$ particle.

The fourth right-side term of (1) is the repulsive velocity component. The role of this component is to push the particle out from the territory of other group's gbest intruded by the particle.

Thanks to the concepts of territory and repulsive velocity, MGPSO could find multiple optima in the solution space. Moreover, MGPSO had a unique advantage over the other algorithms in that one can search $N$ 'superior' peaks of a multimodal function when the number of groups is $N$ [7].

\section{PROPOSED AT-MGPSO}

\section{A. Auto-Tuning Territory}

In MGPSO, the radii of all territories initially having identical size were decreased linearly from $R_{0}$ to zero as the iteration 


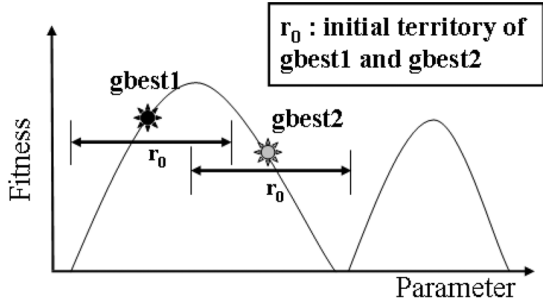

(a)

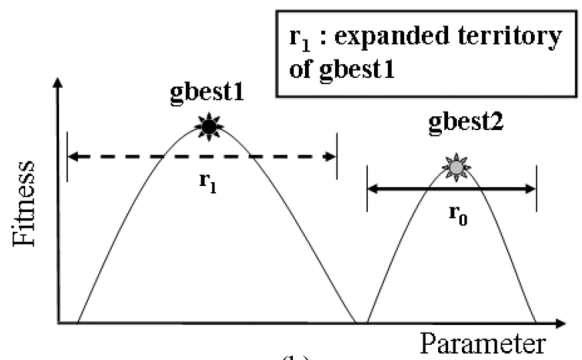

(b)

Fig. 1. A schematic illustration of auto-tuning territory of gbest: 'gbest1' is the gbest of group1. 'gbest2' is the gbest of group2. (a) Before applying auto-tuning territory; (b) after applying auto-tuning territory.

count increased [7], when this decrement rate was set preliminarily by the users. However, the conventional algorithm had a problem: if the sizes of the territory become too small before sufficient convergence level, some groups may not find their own peaks and wander around other groups' gbests, resulting in unnecessary increment of the number of function calls. This phenomenon obviously degraded the efficiency and robustness of the algorithm.

To solve the problem, we propose a new technique to adjust each territory's radius automatically regardless of the iteration count.

1) Competition: Starting from a relatively small initial size of territory, a territory of a group increases its scope when the fitness of the group's gbest is higher than that of its adjacent group's gbest. Two groups can be considered to be 'adjacent' when their territories overlap with each other. The winner group remains and it's radius of territory increases by dividing it by ' 0.95 '. The loser groups are expelled and reinitialized out of the existing groups' territories to search for other peaks in the solution space, which is mimicking a territorial dispute between different groups in ecosystem. The basic idea is such that a peak with a broad scope of influence and high fitness has higher probability to be invaded by other groups because each group has a characteristic of global optimization based on its own gbest.

Simplified explanation on the process is illustrated in Fig. 1, where two groups (group1 and group2) are assumed. The ' $\mathbf{r}_{0}$ ' represents initial territory. Since two territories overlap each other, the region of group1 having better fitness (winner group) is automatically increased to ' $r$ ' ' to preserve its realm from invasions. Loser group (group2) is expelled away from the region and finally finds another peak in space [Fig. 1(b)].

Using this concept, each group's territory size can be controlled automatically and thus one can find other solutions effectively.

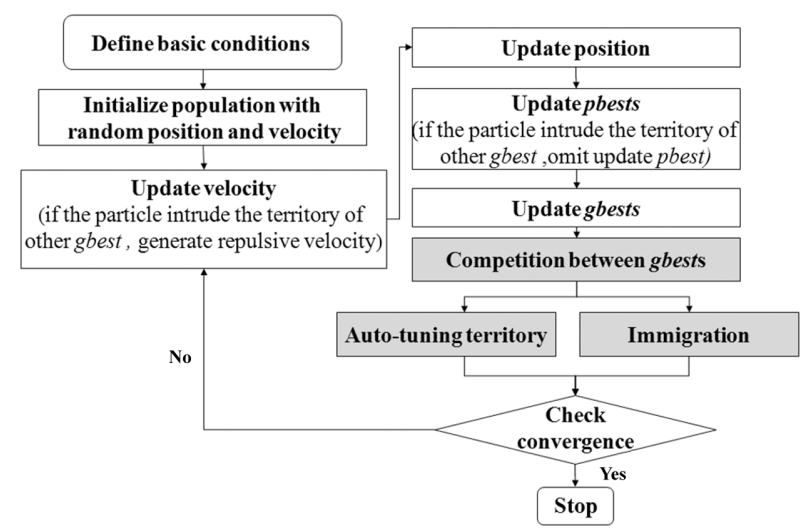

Fig. 2. Flow chart of AT-MGPSO.

2) Initial Radius of Territory: In the conventional MGPSO algorithm, the size of initial territory was set empirically as 5\% of entire solution range. However, it cannot be generally applied to many practical cases since there can be variety of group numbers and solution spaces.

In this paper, we propose an efficient way to determine the initial radius of a territory. Assuming that all $N$ superior peaks are evenly distributed in the solution space $X_{d}$, we decided the initial size of territory $R_{0}$ by the following:

$$
R_{o}=\frac{X / N^{1 / d}}{2} \times 0.7
$$

where $X$ and $d$ are size of solution space and number of dimension respectively. The ' 0.7 ' is an empirical coefficient for the territory size.

\section{B. Immigration of Group}

Since AT-MGPSO increases the size of each group's territory, all particles belonging to a certain group sometimes invade the territories of the other groups. In such a case, we cannot define the gbest of the group because we select the gbest among the solutions which are not included in any other groups' territories. In nature, it is obvious that a species failed to struggle for existence should immigrate to other region for their survival. To mimic the ecological phenomenon and to tackle the potential problem in AT-MGPSO, we removed the 'exterminated' group which could not evaluate its own gbest from the solution space and regenerated a new group out of the existing groups' territories.

The overall flowchart of AT-MGPSO including above explanation is shown in Fig. 2.

\section{Numerical Test AND Result}

To compare the performance of the proposed method with conventional ones such as conventional MGPSO and niching genetic algorithm (Niching GA) [8], a multimodal test function was used. Each simulation consists of 50 iterations.

The formula of the test function, which is a linear summation of single bell-shape functions, is

$$
f=\sum_{k=1}^{N_{p}} \frac{b_{k}}{1+\frac{\left(x-x_{p k}\right)^{2}+\left(y-y_{p k}\right)^{2}}{a_{k}}}, \quad-80<x, y<+80
$$




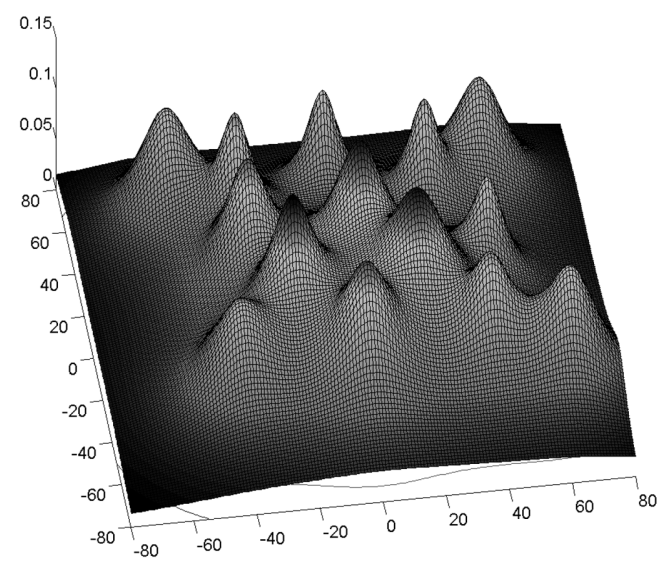

Fig. 3. A test multimodal function.

TABLE I

OPTIMIZATION RESULTS OF TEST FUNCTION

\begin{tabular}{ccc}
\hline \hline & $\begin{array}{c}\text { Success } \\
\text { rate }\end{array}$ & $\begin{array}{c}\text { Number of } \\
\text { function call }\end{array}$ \\
AT-MGPSO & 0.95 & 3055 \\
MGPSO & 0.75 & 4844 \\
Niching GA & 0.44 & 7251 \\
\hline
\end{tabular}

where $N_{p}$ is number of peaks. $a_{k}$ and $b_{k}$ are width and value of $k$ th peak. $x_{p k}$ and $y_{p k}$ are position of $k$ th peak. Fig. 3 shows an example of the test function, where total number of peaks is 14 and all peaks' widths and fitness values are set differently from each other. The total number of groups and particles for both AT-MGPSO and MGPSO is 14 and 15, respectively. And the initial population number of Niching GA is 210 .

Table I presents the simulation results. It is shown that the proposed method reduces the number of function evaluation and has high probability of success, compared to the other methods.

To observe the effect of the proposed technique, we applied the conventional AT-MGPSO to the same test function when the number of groups is 6 . The process of the simulation is depicted in Fig. 4. It can be seen that the proposed AT-MGPSO can find six superior peaks without any scheduling for territory size reduction due to the proposed auto-tuning concept.

Fig. 5 shows the optimization process of the conventional MGPSO. As seen in the figures, two groups are wandered around one dominant peak having high fitness value and broad width. Also they are not converged sufficiently due to the influence of repulsive velocity affecting each other. As a result, the conventional MGPSO miss two superior solutions and waste the computational time.

\section{Application to the Optimal Design of IPMSM}

\section{A. Objective Function and Design Variables}

As a practical optimization example, interior permanent-magnet synchronous motor (IPMSM) for $42 \mathrm{~V}$ Integrated Starter-Generator (ISG) was selected. The initial structure of the analysis model is presented in Fig. 6. The objective of the optimal design to be maximized was defined by

$$
\text { Torque }=\frac{3}{2} \frac{P}{2}\left\{\left(L_{d}-L_{q}\right) i_{d} i_{q}+\lambda_{f} i_{q}\right\}[N . m]
$$

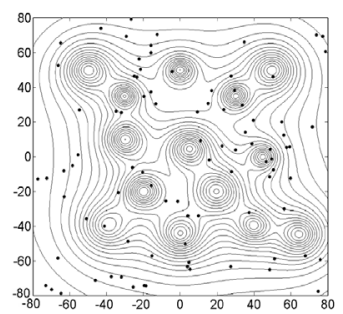

(a)

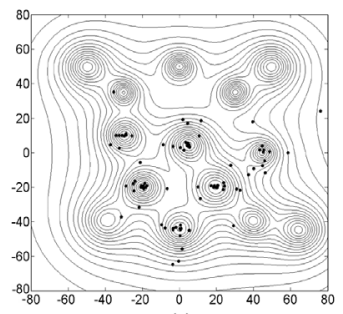

(c)

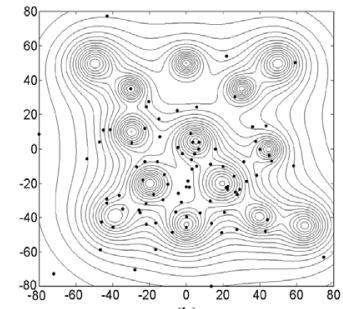

(b)

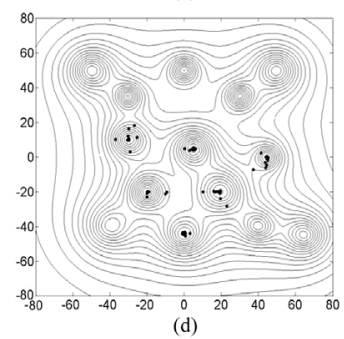

Fig. 4. Optimization result using AT-MGPSO for a test function $(\mu=6$, $\lambda=20$ ). Although some dominant peaks exist, AT-MGPSO can find 6 superior peaks in this solution region. (a) Iteration 0; (b) iteration 10; (c) iteration 20; (d) iteration 30 .
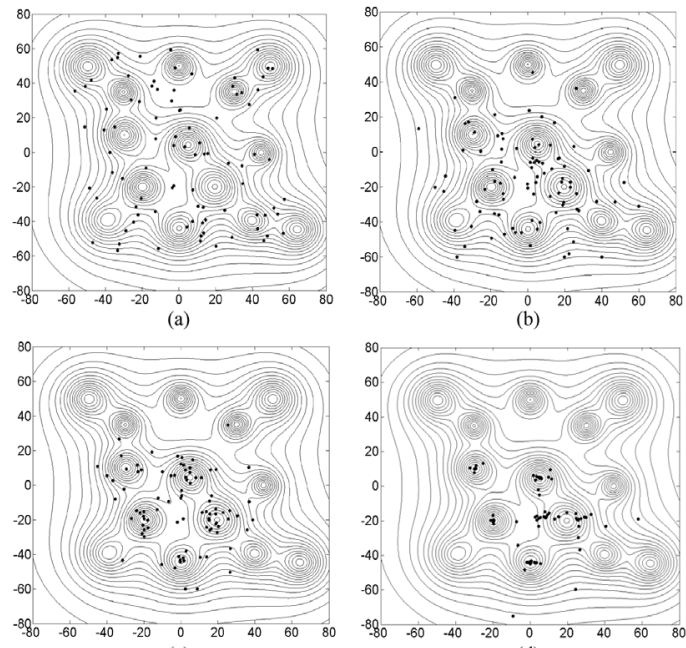

(c)

Fig. 5. Optimization result using MGPSO for a test function $(\mu=6, \lambda=20)$. Because territory of gbest is too small before convergence, two groups are wandering around one peak having high fitness value and broad width. (a) Iteration 0 ; (b) iteration 10; (c) iteration 20; (d) iteration 30 .

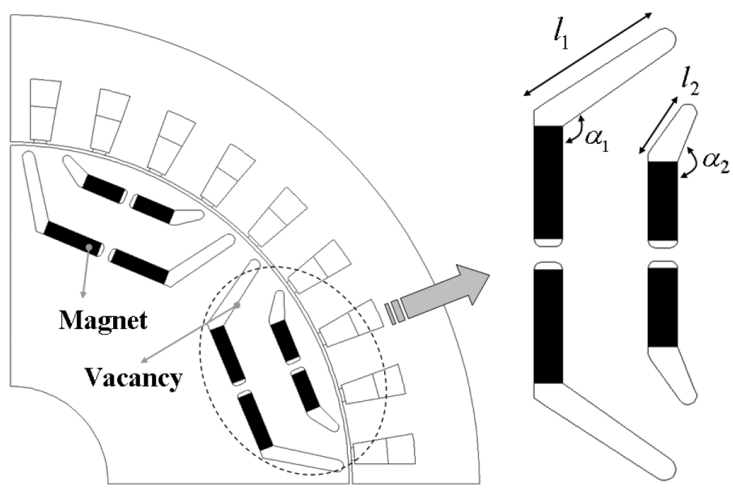

Fig. 6. Structure of an IPMSM $(\alpha 1, \alpha 2)$ : magnet pole arc angle, $\left(l_{1}, l_{2}\right)$ : layer length.

where $L_{d}$ and $L_{q}$ are d- and q-axis inductances, $i_{d}$ and $i_{q}$ are $\mathrm{d}$ - and $\mathrm{q}$ - axis currents, and $\lambda_{f}$ is magnet flux linkage. 
TABLE II

Conditions to Execute the Proposed Algorithm

\begin{tabular}{cc}
\hline \hline Number of variable & 4 \\
Number of total iterations & 100 \\
Number of groups $(\mu)$ & 3 \\
Number of particles in a group $(\lambda)$ & 20 \\
$\mathrm{C}_{1}$ & From 2 to 0.5 \\
$\mathrm{C}_{2}$ & From 2 to 3 \\
$\mathrm{C}_{3}$ & From 4 to 0 \\
\hline
\end{tabular}

TABLE III

SPECIFICATION OF AN OBJECTIVE MOTOR

\begin{tabular}{cc}
\hline \hline Rated power & $3000[\mathrm{~W}]$ \\
Rated RPM & $15000[\mathrm{rpm}]$ \\
Stator outer radius & $154[\mathrm{~mm}]$ \\
Stacking length & $80[\mathrm{~mm}]$ \\
Air gap length & $0.5[\mathrm{~mm}]$ \\
Permanent magnet & Sintered NdFeB \\
\hline
\end{tabular}

If the value of torque at starting is similar, solution with lower total harmonic distortion (THD) of EMF, lower amplitude of EMF and high saliency ratio is preferred. The torque can be evaluated by (5) and thus easily applied to the optimization process; whereas the both THD of EMF and peak of EMF require additional numerical computations and thus is hard to be applied to the optimization process. Therefore, in this study, we first selected several solutions which have high motor torque, and then calculated the THD of EMF and peak of EMF only for the candidate solutions.

To protect inverter devices in case of the fault condition at the maximum speed, magnet quantity should be constrained. Due to the limit condition, we selected four parameters involved in reluctance torque, which has an important role in increasing the starting torque.

If the number of turns, slots, poles, air gap length, stator outer radius and stacking length are fixed on constant values, two magnet pole arc angles $(\alpha 1, \alpha 2)$, two layer lengths $(11,12)$ are taken as design variables. The design variables are shown in Fig. 6. Tables II and III are the basic conditions to execute the MGPSO and the specifications of IPMSM, respectively.

\section{B. Optimization Results}

Table IV shows the three superior solutions optimized for maximum motor torque. Then, the THD of EMF and peak of EMF were evaluated for each candidate solution. From the table, we can see that the case 1 is the best compromise solution because it has sufficiently high starting torque, compared to the other solutions, small EMF, and low THD of EMF.
TABLE IV

OPTIMIZATION RESULTS

\begin{tabular}{cccc}
\hline \hline Solutions & Case 1 & Case 2 & Case 3 \\
Starting torque [Nm] & 74.2 & 74.3 & 73.4 \\
THD of EMF [\%] & 7.2 & 9.5 & 7.5 \\
Amplitude of EMF [V] & 88.5 & 89.9 & 90.4 \\
Saliency ratio & 2.9 & 2.7 & 2.6 \\
$l_{1}[\mathrm{~mm}]$ & 10.3 & 11.2 & 9.2 \\
$l_{2}[\mathrm{~mm}]$ & 3.7 & 2.8 & 2.3 \\
$\alpha_{1}\left[^{\circ}\right]$ & 130 & 128 & 126 \\
$\alpha_{2}\left[^{\circ}\right]$ & 155 & 153 & 143 \\
\hline
\end{tabular}

\section{CONCLUSION}

In the present paper, an improved optimization algorithm named AT-MGPSO was proposed for efficient multimodal function optimizations. Using the new concept of territorial dispute in ecological phenomenon, the AT-MGPSO could find multiple peaks effectively in solution space even where object function is asymmetrical.

The usefulness of AT-MGPSO was verified by the application to a test multimodal function and an optimal design of IPMSM, the results of which demonstrated that the proposed method is promising for multimodal electromagnetic optimization problems.

\section{REFERENCES}

[1] J. Kennedy and R. Eberhart, "Particle swarm optimization," in Proc. IEEE Int. Conf. Neural Networks, 1995, vol. 4, pp. 1942-1948.

[2] K. E. Parsopoulos and M. N. Vrahatis, "On the computation of all global minimizers through particle swarm optimization," IEEE Trans. Evol. Comput., vol. 8, no. 3, pp. 211-224, 2004.

[3] G. Ciuprina, D. Ioan, and I. Munteanu, "Use of intelligent-particle swarm optimization in electromagnetics," IEEE Trans. Magn., vol. 38, no. 2, pp. 1037-1040, 2002.

[4] J. Robinson and Y. Rahmat-Samii, "Particle swarm optimization in electromagnetics," IEEE Trans. Antennas Propag., vol. 52, no. 2, pp. 397-407, 2004

[5] J. J. Liang, A. K. Qin, P. N. Suganthan, and S. Baskar, "Comprehensive learning particle swarm optimizer for global optimization of multimodal functions," IEEE Trans. Evol. Comput., vol. 10, no. 3, pp. 281-295, 2006.

[6] D. Parrott and L. Xiaodong, "Locating and tracking multiple dynamic optima by a particle swarm model using speciation," IEEE Trans. Evol. Comput., vol. 10, no. 4, pp. 440-458, 2006.

[7] J. H. Seo, C. H. Im, C. G. Heo, J. K. Kim, H. K. Jung, and C. G. Lee, "Multimodal function optimization based on particle swarm optimization," IEEE Trans. Magn., vol. 42, no. 4, pp. 1095-1098, 2006.

[8] D. H. Cho, J. K. Kim, H. K. Jung, and C. G. Lee, "Optimal design of permanent-magnet motor using autotuning niching genetic algorithm," IEEE Trans. Magn., vol. 39, no. 3, pp. 1265-1268, 2003.

Manuscript received June 24, 2007. Corresponding author: J.-H. Seo (e-mail: jangho78@elecmech.snu.ac.kr). 